\title{
Instability of Planetary Flows using Riemann Curvature: a Numerical Study
}

\author{
Richard Blender \\ Meteorologisches Institut, Universität Hamburg, Hamburg, Germany
}

(Dated: January 25, 2020)

\begin{abstract}
The instability of ideal non-divergent zonal flows on the sphere is determined numerically by the instability criterion of Arnol'd (1966) for the sectional curvature. Zonal flows are unstable for all perturbations besides for a small set which are in approximate resonance. The sectional curvature scales with $m / \ell$ for large total and zonal wave numbers $\ell$ and $m$ of the perturbations. The planetary rotation is stable and the presence of rotation reduces the instability of perturbations.

PACS numbers: 47.20.-k 47.10.+g 92.60.Bh 47.15.Ki 47.32.-y
\end{abstract}

\section{INTRODUCTION}

Arnol'd formulated ideal two-dimensional hydrodynamics as a geodesic in state space [1, 2]. The dynamics is given by a volume reserving diffeomorphism on a torus (double periodic domain). The torus becomes a Riemannian manifold when the metric given by the kinetic energy is added. The instability of flows can be assessed by the Jacobi equation and a flow is considered to be unstable if the sectional curvature in the Jacobi equation is negative. Arnol'd found that the flow on a torus is unstable and used this to explain the limited predictability of atmospheric flows.

Spherical flows have been considered in $3-7]$. Arakelyan and Savvidy [5] and Dowker and Mo-Zheng [6] derive the sectional curvature in the spherical harmonics basis. Yoshida [7] approached the problem by a transformation of the spherical harmonics. Kambe [8] reviews the method and mentions some hydrodynamic examples. Casetti et al. 9] review the applications with an emphasis on statistical mechanics.

The aim of the present work is to derive numerical results for the instability of nondivergent zonal flows on the sphere. The calculations are based on [5] and [6]. The results are visualized for zonal flows with low total wave numbers which correspond to observations. The planetary rotation is considered by adding a solid body rotation represented by the stream-function $Y_{10}$ to the zonal flow stream-functions. This paper is organized as follows: In Section III the instability of spherical flows is described in terms of the sectional curvature which is based on the Riemann curvature tensor. In Section III the results are presented and in Section [V] the findings are summarized and discussed.

\section{SECTIONAL CURVATURE}

This section summarizes the mathematical properties of non-divergent flows on the sphere and introduces the structure constants to derive the Riemann curvature tensor and the sectional curvature. Explicit expressions for the sectional curvature on the sphere are given in [5] and [6]. Here we follow [5]. The Riemann tensor and the sec- tional curvature derived in $[6]$ can be found in the Appendix. We consider non-divergent flows on the sphere $S^{2}$ with unit radius, which are denoted by SDiff $S^{2}$, the volume preserving diffeomorphisms on the sphere. The coordinates are the latitude $\sigma_{1}=-\cos \theta$, and the longitude $\sigma_{2}=\lambda$; the North Pole is at $\theta=0$.

For the stream-functions $\psi$ and $\eta$ divergence-free vector fields $\mathbf{u}, \mathbf{v}$ are determined by

$$
u^{\alpha}=\varepsilon^{\beta \alpha} \partial_{\beta} \psi, \quad v^{\alpha}=\varepsilon^{\beta \alpha} \partial_{\beta} \eta,
$$

for $\alpha, \beta=1,2$ and the anti-symmetric symbol $\varepsilon^{\beta \alpha}=1$ for $(\beta, \alpha)=(1,2),-1$ for $(2,1)$ and 0 otherwise. The derivative $\partial_{\alpha}$ is with respect to the coordinate $\sigma_{\alpha}$.

A Riemannian manifold is a differentiable manifold with a metric $\langle\mathbf{u}, \mathbf{v}\rangle$ for vector fields $\mathbf{u}$ and $\mathbf{v}$. This is chosen proportional to the kinetic energy,

$$
\begin{aligned}
\langle\mathbf{u}, \mathbf{v}\rangle & =\int \nabla \psi \cdot \nabla \eta \mathrm{d} A \\
& =-\int \psi \nabla^{2} \eta \mathrm{d} A=(\psi, \eta)
\end{aligned}
$$

where the last term defines the metric (, ) for the streamfunctions. The area element is $\mathrm{d} A=\mathrm{d} \sigma_{1} \mathrm{~d} \sigma_{2}=$ $\sin \theta \mathrm{d} \theta \mathrm{d} \lambda$.

An orthogonal basis for the stream-functions on the sphere is given by the spherical harmonics

$$
\begin{gathered}
Y_{\ell m}=C_{\ell m} e^{i m \lambda} P_{\ell}^{|m|}(\cos \theta) \\
C_{\ell m}=(-1)^{m} \sqrt{\frac{2 \ell+1}{4 \pi} \frac{(\ell-|m|) !}{(\ell+|m|) !}}
\end{gathered}
$$

with the Condon and Shortley phases $(-1)^{m}$ not included in the associated Legendre polynomials $P_{\ell}^{|m|}(\cos \theta)$. The spherical harmonics are orthogonal

$$
\left(Y_{\ell_{1} m_{1}}, Y_{\ell_{2} m_{2}}\right)=-(-1)^{m_{1}} \ell_{1}\left(\ell_{1}+1\right) \delta_{\ell_{1}, \ell_{2}} \delta_{m_{1},-m_{2}}
$$

in the kinetic energy metric (2).

A Poisson algebra can be defined for stream-functions $\psi$ and $\eta$

$$
[\psi, \eta]_{P}=\partial_{1} \psi \partial_{2} \eta-\partial_{2} \psi \partial_{1} \eta
$$


with the structure constants $G_{\ell_{1} m_{1} \ell_{2} m_{2}}^{\ell_{3} m_{3}}$

$$
\left[Y_{\ell_{1} m_{1}}, Y_{\ell_{2} m_{2}}\right]_{P}=G_{\ell_{1} m_{1} \ell_{2} m_{2}}^{\ell_{3} m_{3}} Y_{\ell_{3} m_{3}}
$$

Since the structure constants on the sphere are imaginary, it is convenient to use the imaginary parts (the so-called real structure constants) $g$

$$
G_{\ell_{1} m_{1} \ell_{2} m_{2}}^{\ell_{3} m_{3}}=-i(-1)^{m_{3}} g_{\ell_{1} m_{1} \ell_{2} m_{2}}^{\ell_{3}-m_{3}}
$$

Arakelyan and Savvidy [5] derive the real structure constants $g$

$$
\begin{aligned}
& \left(\frac{4 \pi}{\left(2 \ell_{1}+1\right)\left(2 \ell_{2}+1\right)\left(2 \ell_{3}+1\right)}\right)^{1 / 2} g_{\ell_{1} m_{1} \ell_{2} m_{2}}^{\ell_{3} m_{3}}= \\
= & m_{2} \sum_{k_{1}}\left[2\left(\ell_{1}-2 k_{1}-1\right)+1\right] \sqrt{\frac{\left(\ell_{1}-\left|m_{1}\right|\right) \ldots\left(\ell_{1}-\left|m_{1}\right|-2 k_{1}\right)}{\left(\ell_{1}+\left|m_{1}\right|\right) \ldots\left(\ell_{1}+\left|m_{1}\right|-2 k_{1}\right)}}\left(\begin{array}{ccc}
\ell_{1}-2 k_{1}-1 & \ell_{2} & \ell_{3} \\
m_{1} & m_{2} & m_{3}
\end{array}\right)\left(\begin{array}{ccc}
\ell_{1}-2 k_{1}-1 & \ell_{2} & \ell_{3} \\
0 & 0 & 0
\end{array}\right) \\
- & m_{1} \sum_{k_{2}}\left[2\left(\ell_{2}-2 k_{2}-1\right)+1\right] \sqrt{\frac{\left(\ell_{2}-\left|m_{2}\right|\right) \ldots\left(\ell_{2}-\left|m_{2}\right|-2 k_{2}\right)}{\left(\ell_{2}+\left|m_{2}\right|\right) \ldots\left(\ell_{2}+\left|m_{2}\right|-2 k_{2}\right)}}\left(\begin{array}{ccc}
\ell_{1} & \ell_{2}-2 k_{2}-1 & \ell_{3} \\
m_{1} & m_{2} & m_{3}
\end{array}\right)\left(\begin{array}{ccc}
\ell_{1} & \ell_{2}-2 k_{2}-1 & \ell_{3} \\
0 & 0 & 0
\end{array}\right)
\end{aligned}
$$

with the 3 -j symbols $(:::)$.

The instability of a flow $\psi$ with respect to perturbations $\eta$ is determined by the sectional curvature $K(\psi, \eta)$ in the Jacobi equation [2]. In the spherical harmonics basis the two flows are represented as

$$
\psi=\sum_{\ell m} \psi^{\ell m} Y_{\ell m}, \quad \eta=\sum_{\ell m} \eta^{\ell m} Y_{\ell m}
$$

The flows $\psi$ and $\eta$ are normalized, $\langle\psi, \psi\rangle=1,\langle\eta, \eta\rangle=1$, and orthogonal, $\langle\psi, \eta\rangle=0$.

The sectional curvature in the basis of the spherical harmonics is [6]

$$
K(\psi, \eta)=-\sum R_{\ell_{1} m_{1} \ell_{2} m_{2} \ell_{3} m_{3} \ell_{4} m_{4}} \psi^{\ell_{1} m_{1}} \eta^{\ell_{2} m_{2}} \psi^{\ell_{3} m_{3}} \eta^{\ell_{4} m_{4}}
$$

where the sum is over all wave numbers $\ell_{i}$ and $m_{i}, i=$ $1, \ldots, 4$.

In the spherical harmonics basis, the Riemann tensor is determined by the structure constants [5]

$$
\begin{aligned}
R_{\ell_{1} m_{1} \ell_{2} m_{2} \ell_{3} m_{3} \ell_{4} m_{4}} & =\sum_{\ell m}(-1)^{m} \ell(\ell+1) \\
& {\left[d_{\ell_{1} \ell_{3}}^{\ell} d_{\ell_{2} \ell_{4}}^{\ell} G_{\ell_{1} m_{1} \ell_{3} m_{3}}^{\ell m} G_{\ell_{2} m_{2} \ell_{4} m_{4}}^{\ell-m}\right.} \\
& -d_{\ell_{1} \ell_{4}}^{\ell} d_{\ell_{2} \ell_{3}}^{\ell} G_{\ell_{1} m_{1} \ell_{4} m_{4}}^{\ell m} G_{\ell_{2} m_{2} \ell_{3} m_{3}}^{\ell-m} \\
& \left.+k_{\ell_{3} \ell_{4}}^{\ell} G_{\ell_{1} m_{1} \ell_{2} m_{2}}^{\ell m} G_{\ell_{3} m_{3} \ell_{4} m_{4}}^{\ell-m}\right]
\end{aligned}
$$

with

$$
d_{\ell_{1} \ell_{2}}^{\ell}=\frac{1}{2} \frac{\ell(\ell+1)-\ell_{1}\left(\ell_{1}+1\right)+\ell_{2}\left(\ell_{2}+1\right)}{\ell(\ell+1)}
$$

and

$$
k_{\ell_{1} \ell_{2}}^{\ell}=\frac{1}{2} \frac{\ell(\ell+1)-\ell_{1}\left(\ell_{1}+1\right)-\ell_{2}\left(\ell_{2}+1\right)}{\ell(\ell+1)}
$$

In the Appendix the sectional curvature and the Riemann tensor of [6] are quoted.

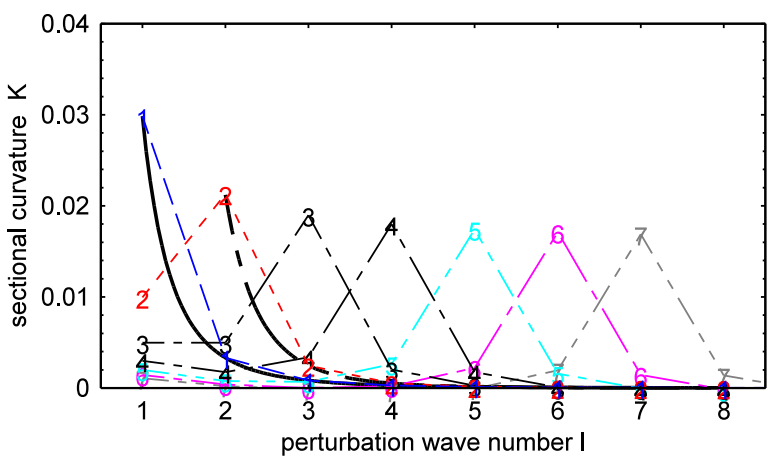

FIG. 1. Sectional curvature $K(\psi, \eta)$ for $\psi=Y_{L 0}$ with $L$ as indicated and perturbations $\eta_{\ell m}$ with $m=1$. Results from [6] are included for $L=1$, Eq. (15), solid-bold, and $L=2$, Eq. (16) for $\ell \geq 2$, dashed-bold.

\section{RESULTS}

The numerical algorithm is first applied to the instability of the zonal flows $Y_{L 0}$ for the reduced set of perturbations $\eta_{\ell m}$ with $m=1$ (Fig. 11). The analysis confirms previous results for $L=1$ and $L=2(\ell \geq 2)[6]$. For example for $L=1$

$$
K=\frac{3}{8 \pi} \frac{1}{\ell^{2}(\ell+1)^{2}}
$$

and for $L=2$

$$
K=\frac{403}{8 \pi} \frac{1}{\ell^{2}(\ell+1)^{2}(2 \ell+3)(2 \ell-1)}, \quad \ell \geq 2
$$

A quasi regular pattern emerges where the stability is highest for perturbations with total wave numbers close to the mean flow, $\ell \approx L$. 
The stability of zonal flows $\psi=Y_{L 0}$ with respect to arbitrary perturbations $\eta=Y_{\ell m}$ is presented in Fig. 22, Note that the results for $m=1$ in Fig. 1 1 are visible along the boundary for low $L$. For higher $L$ this becomes invisible since the instabilities increase and the scale changes accordingly.

Several findings in Fig. 2 are noted:

- The sectional curvatures scales with the argument $m / \ell$ for large wave numbers $\ell, m$. Furthermore, the curvature varies weakly along these lines (see for example $L=3$ ). This property could be useful for expansions and approximations.

- The instability increases with $L$. This supports the dominance of low meridional wave numbers of zonal flows on planets.

- The stability of flows with even and odd total wave numbers differs for the highest $m$ : for odd $L$ the curvature is less negative for high $m$, whereas for even $L$ the instability is most negative for $m=\ell$. Thus flows with odd total wave numbers $L$ (symmetric with respect to the equator) are less unstable.

The above results show that the zonal flow $Y_{10}$ which corresponds to a solid body rotation is stable with respect to all perturbations. Thus planetary rotation might explain the observed stability of zonal slows. Here we consider the superposition of a rotation $Y_{10}$ with weak zonal flows (one order of magnitude smaller). The instabilities in Fig. 3 are reduced by an order of magnitude. Note that the reduction is not a simple rescaling with one magnitude; it depends on $L$ and flows with small $L$ benefit more from the stabilization effect than those with higher $L$.

\section{SUMMARY AND DISCUSSION}

The instability of ideal non-divergent spherical flows is determined numerically by the instability criterion of Arnol'd [1, 2] for the sectional curvature. Using structure constants and the Riemann curvature tensor in a spherical harmonics basis the sectional curvature is determined numerically for stationary zonal flows $Y_{L 0}$ with meridional wave numbers $L$. Zonal flows for $L \geq 2$ are unstable for all perturbations besides for a small set with $m=1$. For perturbations with large total and zonal wave numbers $\ell$ and $m$ the sectional curvature scales with $m / \ell$.

The planetary (solid body) rotation given by $Y_{10}$ is stable. A superposition of intense planetary rotation reduces the instability of zonal flows $Y_{L 0}$ for $L>1$. This result confirms the $\beta$-effect in geophysical fluid dynamics where $\beta$ is the meridional derivative of the Coriolis parameter $f, \beta=d f / d \phi$, with latitude $\phi(\phi=0$ at the equator). The $\beta$-effect sustains Rossby waves and stabilizes geophysical turbulence. Nevertheless, the stabilization is too weak to compensate instabilities even if the
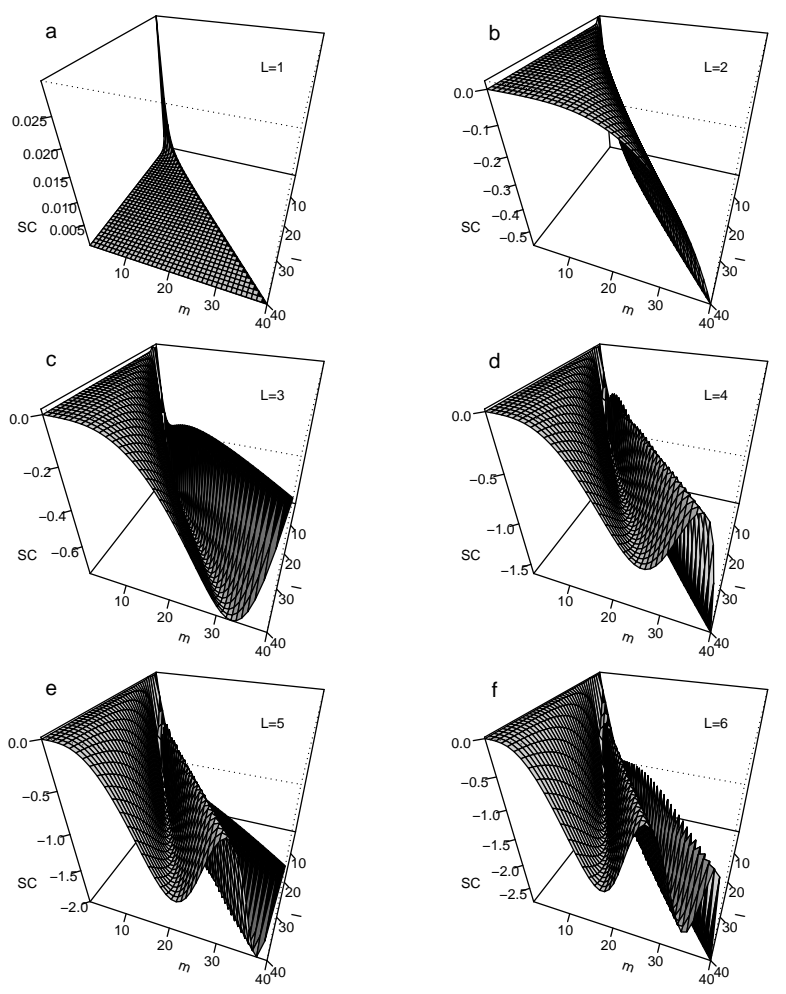

FIG. 2. Sectional curvature $K(\psi, \eta)$ for zonal flows $\psi=Y_{L 0}$ and perturbations $\eta_{\ell m}$. (a) $L=1$, solid body rotation, which is stable for all perturbation wave numbers $(\ell, m)$. (b)-(f) Sectional curvature for increasing total wave numbers $L=$ $2, \ldots, 6$ as indicated.

rotation is one order of magnitude more intense than the zonal flows.

This study cannot explain the observed dominance of zonal flows on planets. There are two main reasons: A first is that the assumption of a non-divergent ideal flow is too simplistic. Realistic flow patters need to be simulated with complex geophysical models (see for example [10]). A second reason might be that the interpretation of the sectional curvature as an indicator for instability is not yet fully understood (chaos can, for example, be induced by fluctuations of positive curvatures as well [9]).

\section{ACKNOWLEDGMENTS}

These results have been presented at the EGU 2011, Vienna, and during the Workshop on Instabilities and Fluctuations of Geophysical Flows in Hamburg, June 46, 2014, funded by the Deutsche Forschungsgemeinschaft.

\section{Appendix A: Riemann tensor by Dowker and Mo-zheng (1990)}

Dowker and Mo-zheng [6] determine an alternative closed expression for the negative imaginary part of the 

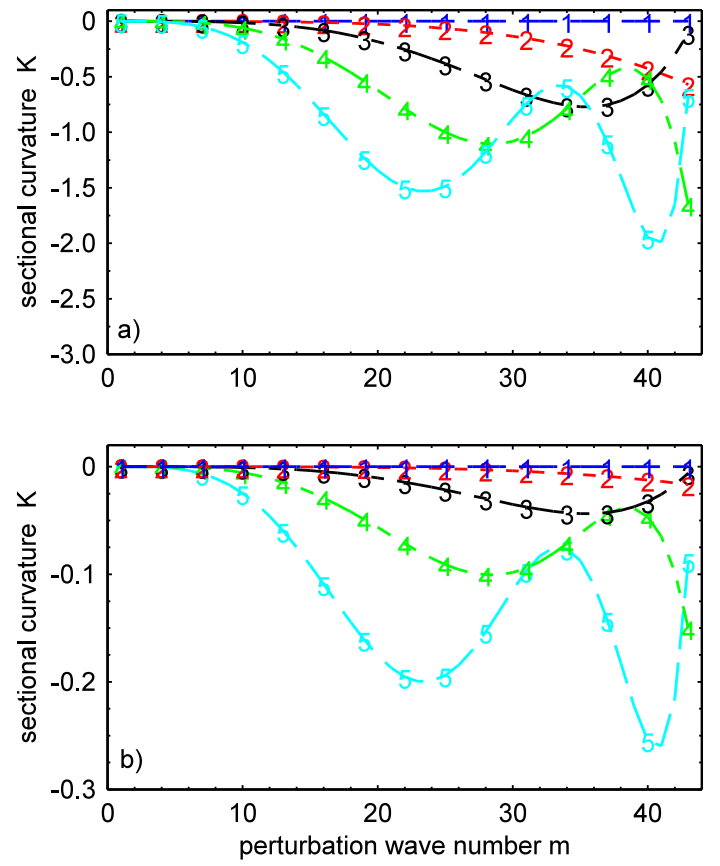

FIG. 3. Impact of rotation: a) Sectional curvature $K(\psi, \eta)$ for zonal flows $\psi=Y_{L 0}, L=1, \ldots, 5$, versus the zonal wave number $m$ of the perturbations $\eta^{\ell m}$ with constant $\ell=43, \mathrm{~b}$ ) with solid body rotation superimposed to weak zonal flows, $\psi=Y_{10}+0.1 Y_{L 0}$.

structure constants (8). These results are quoted (with a small correction) since they turned out as numerically efficient.

$$
\begin{aligned}
& G_{\ell_{1} m_{1} \ell_{2} m_{2}}^{\ell_{3} m_{3}}=-i C_{m_{1} m_{2} m_{3}}^{\ell_{1} \ell_{2} \ell_{3}} \\
& C_{m_{1} m_{2} m_{3}}^{\ell_{1} \ell_{2} \ell_{3}}=\frac{(-1)^{m_{3}}}{\sqrt{4 \pi}} L_{123}\left(\begin{array}{ccc}
\ell_{1} & \ell_{2} & \ell_{3} \\
m_{1} & m_{2} & -m_{3}
\end{array}\right) \\
& \times\left(\begin{array}{ccc}
\ell_{1} & \ell_{2} & \ell_{3} \\
1 & -1 & 0
\end{array}\right)
\end{aligned}
$$

with

$$
L_{123}=\left[\left(2 \ell_{1}+1\right)\left(2 \ell_{2}+1\right)\left(2 \ell_{3}+1\right) \ell_{1}\left(\ell_{1}+1\right) \ell_{2}\left(\ell_{2}+1\right)\right]^{1 / 2}
$$

The Riemann curvature components are in the notation of [6]

$$
\begin{aligned}
R_{m_{1} m_{2} m_{3} m_{4}}^{\ell_{1} \ell_{2} \ell_{3} \ell_{4}} & =\sum_{\ell m}(-1)^{m} \ell(\ell+1) \\
{[} & -d_{\ell_{1} \ell_{3}}^{\ell} d_{\ell_{2} \ell_{4}}^{\ell} C_{m_{1} m_{3} m}^{\ell_{1} \ell_{3} \ell} C_{m_{2} \ell_{4}-m}^{\ell_{2} \ell_{4} \ell} \\
& +d_{\ell_{1} \ell_{4}}^{\ell} d_{\ell_{2} \ell_{3}}^{\ell} C_{m_{1} m_{4} m}^{\ell_{1} \ell_{4} \ell} C_{m_{2} m_{3}-m}^{\ell_{2} \ell_{3} \ell} \\
& -k_{\ell_{3} \ell_{4}}^{\ell} C_{m_{1} m_{2} m}^{\ell_{1} \ell_{2} \ell} C_{m_{3} m_{4}-m}^{\ell_{3} \ell_{4} \ell}
\end{aligned}
$$

Note that [6] mention a different expression (possibly a typo); the relationships (A1A2 were verified numerically with (8) and (9).
[1] V. Arnol'd. Ann. Inst. Fourier, 16:319, 1966.

[2] V. Arnol'd. Mathematical Methods of Classical Mechanics. Springer-Verlag, Berlin, 1978.

[3] A. M. Lukatskii. Curvature of groups of diffeomorphisms preserving the measure of the 2-sphere. Functional Anal. Appl., 13:174-178, 1979.

[4] A. M. Lukatskii. Structure of the curvature tensor of the group of measure-preserving diffeomorphisms of a compact two-dimensional manifold. Siberian Math. J., 29:947-951, 1988.

[5] T. A. Arakelyan and G. K. Savvidy. Geometry of a group of area-preserving diffeomorphisms. Physics Letters B, 223:41-46, 1989.
[6] J. S. Dowker and W. Mo-zheng. Area-preserving diffeomorphisms and the stability of the atmosphere. Class. Quantum Grav., 7:2361-2365, 1990.

[7] K. Yoshida. Riemann curvature on the group of areapreserving diffeomorphisms (motions of fluid) of 2-sphere. Physica D, 100:377-389, 1997.

[8] T. Kambe. Geometrical aspects in hydrodynamics and integrable systems. Theoret. Comput. Fluid Dynamics, 10:249261, 1998.

[9] L. Casetti, M. Pettini, and E. G. D. Cohen. Geometric approach to Hamiltonian dynamcis and statistical mechanics. Phys. Reports, 337:237-341, 2000.

[10] Y. Lian and A. P. Showman. Generation of equatorial jets by large-scale latent heating on the giant planets. Icarus, 207(1):373 - 393, 2010. 